\title{
2||-||||||||||||||||||||||||||||||||||||||||||||||||||||||||||||||.
}

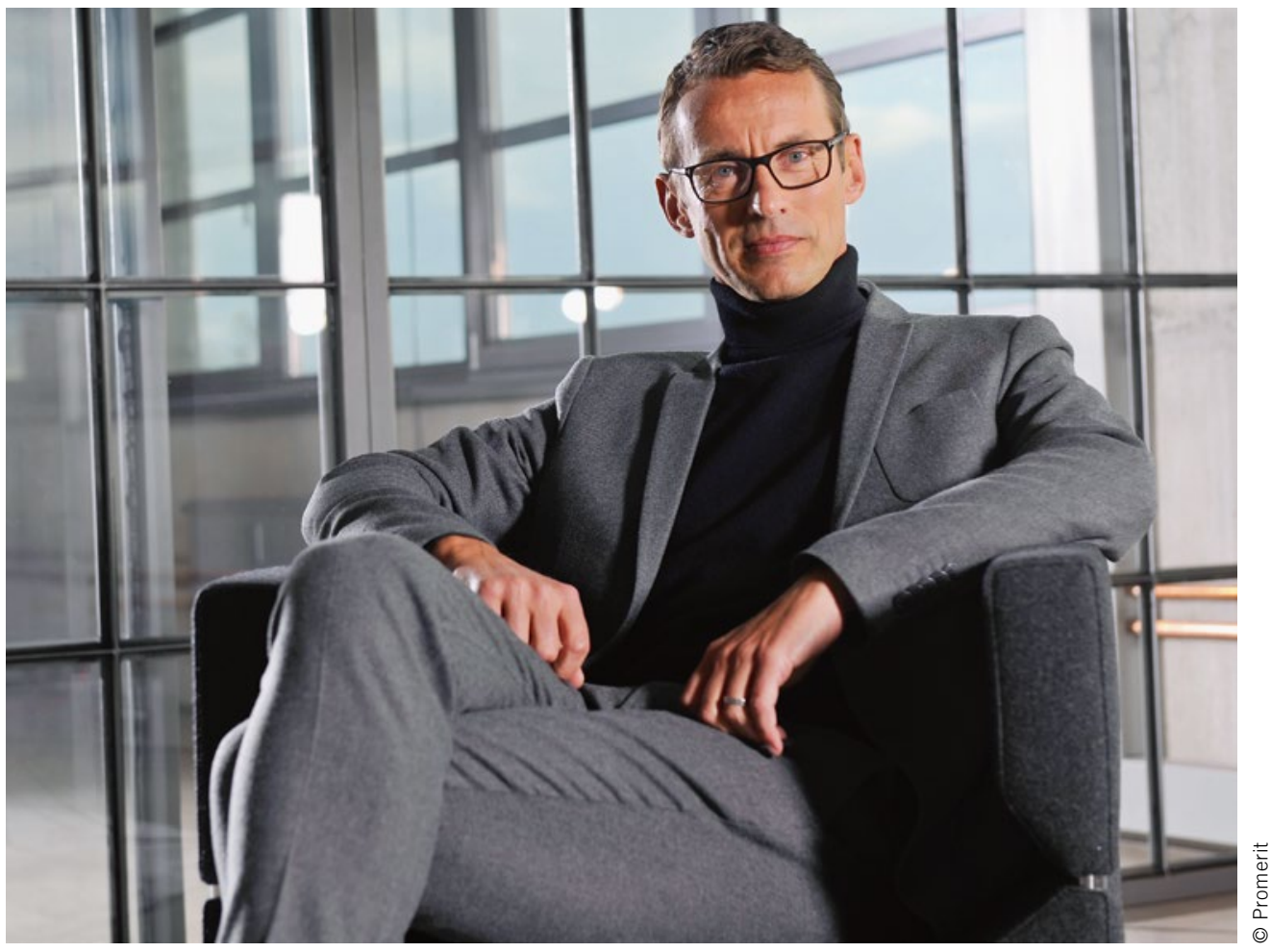

Kai Anderson

Co-founder and Member

of the Board of Promerit AG

\section{An Agile Approach to the Digital Transformation}

Digitization is bringing dramatic changes in all areas of life and presenting businesses with huge challenges. Only a few years ago some industries, in particular those that had had little to do with IT, the cloud, and the Internet, did not seem to be fully aware of the inevitability of this. The car industry began working at a fairly early stage on autonomous driving systems and infotainment modules, which were aimed primarily at distracting passengers and eventually also drivers with entertainment and new opportunities for working in cars. But only a few people in the automotive industry considered calling the entire system of individual transport into question. Now the lead strategists at the carmakers are working on plans for software apps like the one used by Uber that will revolutionize personal transport and turn it into a commodity service which can be used anywhere at any time, without the need to own a car. No driver, no parking, no congestion. Is this a Utopian vision of the future? No. This scenario is likely to become reality in many megacities between 2030 and 2035.

This puts the business model of the car manufacturers in doubt and at the moment the German automotive industry is not a strong position in this respect. We need to ensure that the future mobility platforms do not come only from the USA and Asia. Another aspect of life that digitization is changing is our view of work and the workplace. The clear boundary between the office and home is becoming blurred because of the growth of home offices. New working methods are increasingly transforming our everyday work routines. The widespread use of Kanban and Scrum in the automotive industry means that it is on the right track. Design thinking is gradually making its way from the IT department into the car development process, despite the fact that the process leaves little leeway for hybrid structures. The automotive industry, with its complex supply chains, is also making good progress toward the networked economy, although the risk of faults is still too high throughout the entire system. But what we are really lacking is a culture that will replace the one we are much too fond of describing as "having gasoline flowing through our veins." This expression is synonymous with a moment of inertia in the last century that we need to get over as quickly as possible. We are no longer the most important, most respected, most attractive industry in the world. It is difficult to take on board, but the German automotive industry needs to reinvent itself in the era of digitization. This will only work if we take a very careful look at what has made us successful in the past. If we understand the essence of digitization not only from a technical perspective, but also as the idea of rethinking our products, services, processes, and rules for the benefit of our customers, then we will be moving in the right direction. Ladies and gentlemen, start your digital engines! 